\title{
Origins of the cytolytic synapse
}

Maike de la Roche* Yukako Asano and Gillian M. Griffiths

Cambridge Institute for Medical Research, University of Cambridge, CB2 OXY, UK.

*Present address: CRUK-Cambridge Research Institute, Cambridge, CB2 ORE, UK.

Correspondence to G.M.G. e-mail: gg305@cam.ac.uk

or M.D.R.: maike.delaroche@cruk.cam.ac.uk

\begin{abstract}
Cytotoxic T lymphocytes (CTLs) kill virus-infected and tumour cells with remarkable specificity. Upon recognition, CTLs form a cytolytic immune synapse with their target cell and marked reorganization of both the actin and microtubule cytoskeletons brings the centrosome right up to the plasma membrane to the point of T cell receptor (TCR) signalling. Secretory granules move towards the centrosome and are delivered to this focal point of secretion. Such centrosomal docking at the plasma membrane also occurs during ciliogenesis; indeed, striking similarities exist between the cytolytic synapse and primary cilium that throw light on the possible origins of immune synapses.
\end{abstract}

\section{Introduction}

Cytotoxic T lymphocytes (CTLs) are a crucial component of the adaptive immune system that function to eliminate intracellular pathogens and tumour cells. They originate from naïve $\mathrm{CD}^{+} \mathrm{T}$ cells in a process termed effector $\mathrm{T}$ cell differentiation.

Naïve $\mathrm{CD}^{+} \mathrm{T}$ cells are small, relatively quiescent cells that circulate between the lymphatic system and blood in search of their specific antigen. Once they encounter antigen presented by MHC class I molecules on the surface of professional antigen-presenting cells (APCs) in the peripheral lymphoid organs, naïve $\mathrm{CD}^{+} \mathrm{T}$ cells slow down and stop - a step that is controlled by integrin signalling ${ }^{1}$ - and $\mathrm{T}$ cell receptor (TCR) activation triggers the formation of a highly specialized cell-to-cell contact, known as the immune 
synapse. This initial 'signalling' synapse induces rapid clonal expansion and effector differentiation and over the next 4-5 days 'unarmed' naïve $C D 8^{+} \mathrm{T}$ cells differentiate into heavily 'armed' effector CTLs loaded with specialized cytolytic granules containing perforin and granzymes. Simultaneously, these small, round naïve $\mathrm{CD} 8^{+} \mathrm{T}$ cells $(\sim 5 \mu \mathrm{m}$ diameter) increase in size (to $\sim 10 \mu \mathrm{m}$ diameter) and develop a much more sophisticated cytoskeletal apparatus, which is required to deliver cytolytic granules to the immune synapse. Once CTLs recognize their target cells in the periphery, a synapse is formed, and the cytolytic granules are secreted at the synapse within minutes.

Structurally, the synapses formed by naïve $\mathrm{CD}^{+} \mathrm{T}$ cells and fully differentiated CTLs with their APCs are very similar: both cell types form a central supramolecular activation cluster (CSMAC) of TCRs ${ }^{2,3}$, polarize their centrosomes (the only type of microtubule-organizing centre (MTOC) in T cells) to the contact site with the APC and reorganize their actin cytoskeleton forming a distal SMAC (dSMAC) ${ }^{4,5}$. However, the purpose of these synapses is very different: naïve $\mathrm{CD} 8^{+} \mathrm{T}$ cells become primed to proliferate and differentiate into CTLs over the course of several days, whereas CTLs are triggered to undergo rapid, polarized secretion of cytolytic granules towards the point of TCR signalling within minutes.

Recent findings have revealed not only the order of many of the early events of synapse formation but also novel mechanisms involved that throw light on the origins of the immune synapse. This opens new avenues for understanding how secretion at the immune synapse is controlled.

\section{Early events in synapse formation}

Initial cell contact

Early electron microscopy studies suggested that the initial interaction between $T$ cells and target cells occurs via projections from the $T$ cell that form an interdigitated contact area between cells ${ }^{6-8}$. Although CTL conjugates formed after 10 minutes do show an interdigitated interface between $T$ cells and target cells, conjugates formed after 60 minutes show extended areas of 
tight membrane contact between the $\mathrm{T}$ cell and target, with membranes separated by a distance of $5-25 \mathrm{~nm}{ }^{6}$, which is consistent with the $13 \mathrm{~nm}$ distance imposed by TCR binding to an MHC molecule ${ }^{9}$. Similarly, T cell projections predominate in early synapses formed by $\mathrm{CD}^{+} \mathrm{T}$ cells ${ }^{10}$. However, the molecular changes underlying the transition from interdigitated to flattened membranes at the synapse are poorly understood, in part because it has been difficult to capture synapse formation at early stages. The use of a genetic model in which the catalytic activity of the TCR signalling protein ZAP70 can be blocked with a small molecule inhibitor to leave only integrin signalling intact has shed light on this phenomenon ${ }^{11}$. In the absence of ZAP70 catalytic activity, synapse formation was arrested at a very early stage after contact with the target cell, with actin-rich interdigitations forming the contact points between the two cells and constituting what appears to be an initial accumulation of actin across the synapse (Figure 1a). However, when ZAP70 is catalytically active, the synapse structure changes markedly to form an extended area of tight membrane contact between $\mathrm{T}$ cell and target cell. The loss of actin-rich interdigitations between the cells correlates with light microscopy images showing an equally marked reduction in the amount of actin across the synapse (Figure 1b). These results point towards an important role for actin reorganization during the initial stages of synapse formation and suggest that fundamental changes occur in the membranes forming the synapse once TCR signalling is initiated.

\section{TCR clustering}

The initial events of TCR signalling have been studied extensively and a great deal has been learnt from studies carried out on artificial planar synapses using total internal reflection fluorescent (TIRF) microscopy ${ }^{12}$. These studies showed that as TCR signalling is initiated, TCR microclusters (TCR-MC) coalesce in the centre of the synapse to form the CSMAC ${ }^{13}$. The formation of the CSMAC has been shown to depend not only on F-actin flow, with inhibitors of F-actin including latrunculin-A and jasplakinolide preventing TCR clustering ${ }^{14-16}$, but also dynein-motor-driven microtubule movement ${ }^{17}$. These observations led to a model in which F-actin flow regulates TCR-MC movement in the periphery of the synapse, whereas dynein-mediated 
transport of TCR-MC along microtubules might occur in the center of the synapse. Both dynein and the microtubule plus-end-binding protein 1 (EB1), which recruits dynein to the plus ends of microtubules, have been found to coimmunoprecipitate the TCR complex component CD3, and depletion of either dynein or EB1 disrupted downstream TCR signalling after stimulation ${ }^{17,18}$. As dynein has a well-established role in vesicle movement, these results support the idea that vesicle-mediated delivery of TCRs to the synapse is important for signalling. This is supported by studies showing an important role for vesicle-mediated transport of TCR-associated signalling proteins to and from the immune synapse, with LCK, LAT and TCR $\zeta$ being delivered in discrete vesicles ${ }^{19-23}$. This easily regulated delivery to and/or removal from the synapse of TCR-associated proteins may provide a mechanism to fine-tune TCR signalling.

\section{Sequence of events}

One problem in trying to create a clear model of the mechanisms leading to signalling and secretion at the cytolytic synapse has been in identifying the order in which events occur. Our recent study using 4D high-resolution imaging of fluorophore-tagged proteins to follow events from signalling to secretion at the cytolytic synapse has led us to propose an integrated model of synapse formation (Figure 2) ${ }^{24}$. From this study, it was possible to determine the timing of actin reorganization, formation of the CSMAC, polarization of the centrosome and delivery of the granules to the synapse in TCR-transgenic CTLs. This study showed that in the first 20s after contact is made between a CTL and target cell, actin accumulates at the synapse, but within the next 20-40s actin depletes across the synapse as the membranes reorganize to form the tight contacts seen by electron microscopy. Concurrent with this reorganization, a pool of plasma membrane-associated TCRs clusters at the centre of the actin-depleted area, forming the CSMAC within 2 minutes of contacting the target cell. Once this has occurred, the centrosome begins to move from the rear of the cell, arriving at the synapse 6 minutes after initial contact and bringing with it a second pool of vesicle-associated

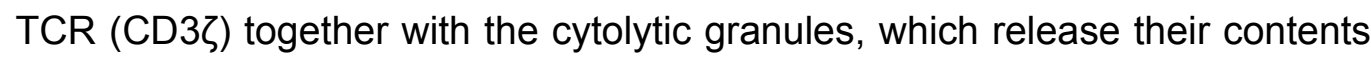
into the synaptic cleft and destroy the target cell. The order of these events 
supports the idea that there are two waves of TCR delivered to the synapse - the first occurs very rapidly as the membrane flattens and actin depletes across the synapse, the second occurs as the centrosome arrives at the synapse immediately before secretion.

\section{Actin flow}

Other surprises emerged from the 4D imaging study ${ }^{24}$. The improved temporal and spatial resolution of lattice light-sheet imaging revealed a rearward flow of actin in both migrating and synapsing CTL, with actin continuing to flow rearward away from the synapse once a target is encountered (Figure $\mathbf{3 a}, \mathbf{b})$. Importantly live imaging shows how rapidly actin reorganization occurs as the cytolytic synapse forms and highlights the difficulties in interpreting fixed images captured at a single point in time. One major change involves the loss of lamellipodial projections from the centre of the forming synapse, which depletes the level of actin in this region to $\sim 20 \%$ of that of the rest of the cell body, while there is an enrichment of actin around the periphery of the synapse where lamellipodial formation becomes focused. These changes reflect a change in the composition of the T cell membrane with a loss of phosphatidylinositol 4,5 bisphosphate $\left(\mathrm{PIP}_{2}\right)$ from the membrane ${ }^{24}$, a phosphoinositide that is required for the recruitment of actinpolymerizing proteins including the ARP2/3 complex ${ }^{25,26}$, consistent with the loss of cortical actin in this region (Figure 3c,d).

Actin flow as the synapse forms is thought to be important in creating the mechanical force required to activate the integrin LFA-1 to its high-affinity form and ensure tight adhesion between $\mathrm{T}$ cell and APC as they meet ${ }^{27}$. These initial contacts occur via actin-rich protrusions at the leading edge of migrating $\mathrm{T}$ cells, which are regulated by protein kinase $\mathrm{C} \delta(\mathrm{PKC} \delta), \mathrm{RAC} 1$ and downstream effectors of actin polymerization including Wiskott Aldrich syndrome protein (WASP) ${ }^{28}$. These protrusions form the first point of contact between cells as synapses form, and they may correspond to the actin foci that have been reported to be required for the activation of PLC $\gamma 1$, via WASP 29. This study has thrown some light on the role of WASP in synapse 
formation, which seems to be important in generating dynamic F-actin structures and thereby amplifying downstream TCR signalling. Thus it emerges that the continual formation and flow of actin-rich projections at the synapse are crucial for both adhesion and effective signalling.

\section{Mechanisms controlling centrosome polarization}

TCR activation not only triggers actin reorganization, but also initiates polarization of the centrosome and associated secretory organelles to the immune synapse (Figure 4). Pinpointing the signalling event that triggers centrosome polarization has been challenging as disruption of proximal TCR signalling kinases, including LCK and ZAP70, and the adaptor protein SLP76, which links proximal TCR stimulation to downstream signalling, all disrupt centrosome polarization to the synapse ${ }^{30-32}$. Further downstream of TCR activation, phospholipase $\mathrm{CY}_{\mathrm{Y}}$ (PLCY) is recruited to the plasma membrane and hydrolyzes $\mathrm{PIP}_{2}$, yielding two second messengers - inositol trisphosphate $\left(\mathrm{IP}_{3}\right)$, which activates $\mathrm{Ca}^{2+}$ signalling, and diacylglycerol (DAG), which accumulates at the synapse ${ }^{33}$. The role of $\mathrm{Ca}^{2+}$ in centrosome polarization is under debate ${ }^{32,34-36}$ but DAG has been shown to be crucial for centrosome polarization ${ }^{36}$. This is thought to occur by a mechanism involving the recruitment of novel $\mathrm{PKC}$ isoforms (PKCס, $\mathrm{PKC} \varepsilon, \mathrm{PKC} \eta$ and $\mathrm{PKC} \theta$ ) that are specifically activated by DAG. PKC $\theta$ facilitates centrosome polarization by reciprocally localizing dynein to the synapse, which pulls the centrosome forward, and nonmuscle myosin II (NMII) to the opposite side of the cell, where it pushes the centrosome towards the synapse ${ }^{37,38}$. Another TCRactivated, DAG-independent atypical PKC (aPKC) isoform, $\mathrm{PKC} \zeta$, which forms part of the partitioning defective (PAR) polarity complex, has been implicated in T cell polarity ${ }^{39,40}$, and work on the TCR-activated PAR family member PAR1B has revealed its role in centrosome polarization in T cells ${ }^{41}$.

Identifying the mechanisms that move the centrosome from the rear of a migrating $\mathrm{T}$ cell to the synapse is complicated by the many proteins that contribute, but the model that is emerging is similar to that described in migrating fibroblasts, in which the centrosome polarizes towards sites of wound healing. With the observation that the minus end-directed microtubule 
motor protein dynein is recruited to the dSMAC ${ }^{42}$ downstream of DAG ${ }^{36}$ and controls centrosome translocation ${ }^{42,43}$, it was suggested that dynein pulls microtubules from the periphery of the synapse by means of a cortical sliding mechanism to translocate the centrosome to the activation site ${ }^{42,44}$. By contrast, another study ${ }^{35}$ suggests that centrosome translocation to the synapse occurs via a capture-shrinkage mechanism in which pioneer microtubules that extend between the centrosome and plasma membrane at the immune synapse use the motor force of dynein together with depolymerization to shorten, and thereby reel in the centrosome in to the synapse. In support of this combination of mechanisms, taxol (which inhibits microtubule depolymerization) and ciliobrevin (a dynein inhibitor) blocked centrosome polarization when used together, whereas taxol alone slowed polarization. Interestingly, pioneer microtubules, which have a role in the formation of protrusions at the leading edge of migratory cells, are regulated by RAC1, stathmin and formins ${ }^{45,46}$, all of which have been shown to be involved in centrosome polarization ${ }^{47-49}$. Consistent with a role for microtubule dynamics in centrosome polarization, depletion of casein kinase $1 \delta$ (CK1 $1 \delta)$, which phosphorylates the microtubule plus-end-binding protein EB1, not only inhibits microtubule stabilization and growth, but also centrosome polarization in T cells ${ }^{50}$.

Studies tracking the speed of centrosome polarization reveal that it is a twostep process, with an average speed of $3.3 \mu \mathrm{m}$ per second as the centrosome approaches the synapse initially, slowing to $0.9 \mu \mathrm{m}$ per second within the final $\sim 2 \mu \mathrm{m}$ from the synapse 24,35 . Intriguingly, in CTLs lacking LCK the centrosome polarises to within $\sim 2 \mu \mathrm{m}$ of the synapse but does not dock at the $\mathrm{T}$ cell membrane, which is consistent with a two-step mechanism for centrosome polarization ${ }^{30}$.

Insights from NK cells

Natural killer (NK) cells offer some unique opportunities for understanding the signals that mediate centrosome polarization. Although NK cells polarize their centrosome in a similar manner to CTLs ${ }^{5,51}$, integrin activation alone triggers 
centrosome and granule polarization in NK cells but not CTLs ${ }^{52-54}$. The TCRassociated signalling molecule ZAP70 is required for integrin activation in T cells; blocking the kinase activity of ZAP70 prevents downstream TCR signalling, but still enables integrin activation ${ }^{55}$. Using this model to examine centrosome polarization in CTLs revealed that integrin signalling in the absence of TCR signalling was not sufficient to polarize the centrosome or cytolytic granules to the synapse in CTLs ${ }^{11}$.

The downstream integrin signalling pathways that control centrosome and granule polarization in NK cells have been identified using a mass spectrometry approach ${ }^{56}$. This study showed that the integrin signal in NK cells is mediated via integrin-linked kinase (ILK), which forms a heterotrimeric complex (the IPP complex) with PINCH1 and $\gamma$-parvin that is known to be involved in cell adhesion, spreading and polarity ${ }^{57}$. Knock-down of either ILK or $\gamma$-parvin inhibited centrosome and granule polarization, without affecting NK cell-target cell conjugate formation ${ }^{56}$. This proteomic approach revealed links to the CDC42-PAR6 network via RhoGEF7, a CDC42 activator, together with the previously identified proteins paxillin and PYK2 ${ }^{58-60}$; inhibition of any of these proteins prevented both centrosome and granule polarization. Given that two phases of centrosome polarization to the synapse have been identified in T cells ${ }^{24,35}$, it will be interesting to discover whether the integrinmediated signals identified in NK cells control one or both steps in centrosome polarization.

Studies of patients with primary immunodeficiencies continue to identify proteins involved in NK cell granule secretion. Patients with mutations in dedicator of cytokinesis 8 (DOCK8) have low T cell numbers ${ }^{61}$, whereas their NK cells have reduced cytotoxicity ${ }^{62}$. Loss of DOCK8 results in reduced integrin-mediated adhesion and defective actin accumulation at the NK cell synapse, with a concomitant reduction in granule polarization ${ }^{62,63}$. The molecular basis of these defects arises from the interaction of DOCK8 with WASP and talin ${ }^{63}$, which are important regulators of actin turnover. More recently a novel DOCK8-interacting protein, Hook-related protein 3 (HKRP3; 
also known as CCDC88B) has been identified that can bind directly to microtubules and can immunoprecipitate dynein from NK cell lysates ${ }^{64}$. Interestingly, depletion of HKRP3 did not affect NK cell synapse formation, but inhibited both centrosome polarization and the clustering of granules, which is consistent with a role for HKRP3 in the microtubule-mediated movement of both centrosome and granules.

Another unique opportunity afforded by NK cells has been to address the role of coronin 1A, an actin regulatory protein - loss of which disrupts $\mathrm{T}$ cell development ${ }^{65}$ - in NK cell cytolytic function. Patients lacking coronin $1 \mathrm{~A}$ have a profound loss of NK cell activity that has been linked to failure to reorganize the synaptic cortical actin, thereby inhibiting granule release ${ }^{66}$. Two studies in NK cells have revealed a fine meshwork of cortical actin across the synapse, and proposed that small holes in this meshwork form to allow the secretion of granules ${ }^{67,68}$. As images of the actin meshwork came from fixed samples rather than live imaging, it is not clear at what point in the series of events leading to granule secretion they might have been taken (Figure 2). 4D imaging of the CTL synapse suggests that an actin meshwork appears just after secretion, rather than before ${ }^{24}$. However it remains possible that actin-mediated regulation of secretion might differ in CTLs and NK cells, although it is clear that the centrosome directs granules to the point of secretion in both cell types ${ }^{5,51}$.

\section{The immune synapse and primary cilium}

Contact between the centrosome and the plasma membrane normally only occurs during ciliogenesis. One of the most striking features of electron micrographs of the centrosome at the immune synapse is its similarity to sites of primary cilium formation. In images of both the synapse and sites of ciliogenesis, it is possible to see not only the centrosome contacting the plasma membrane, but also the associated Golgi apparatus and endocytic recycling compartment polarized towards the plasma membrane at the site of centrosome contact (Figure 5). Lymphocytes are one of the very few cell types that have been reported not to be able to form primary cilia ${ }^{69,70}$. The marked similarities between the immune synapse and primary cilium, first 
noted when centrosome docking at the synapse was initially observed ${ }^{5,71}$, have been of great interest and led to our proposal that the synapse might be a 'frustrated' or modified primary cilium ${ }^{72}$.

The primary cilium is an ancient organelle assembled by almost all nucleated eukaryotic cells between cell divisions. For many years, the primary cilium was regarded as a vestigial organelle without function. However, we now know that the primary cilium is a sensory organelle that integrates multiple signals from the environment into a cellular response ${ }^{73}$. This discovery highlighted possible functional similarities with the immune synapse, which also forms a platform for signalling. How far do these similarities extend and do they have a molecular basis?

\section{Molecular and functional similarities}

Many similarities between the immune synapse and cilium have now emerged (Box 1). In addition to both structures having a membrane-docked centrosome, there are further intriguing structural similarities. For example, cortical actin is reduced in the region where the centrosome attaches at the plasma membrane in both primary cilia ${ }^{74}$ and immune synapses ${ }^{24}$, and a peripheral actin ring delineates a specialized membrane domain within which $\mathrm{PIP}_{2}$ depletion is required for ciliary signalling ${ }^{75-77}$.

The intraflagellar transport (IFT) proteins, which are essential for the assembly and maintenance of cilia and flagella ${ }^{78}$ and were originally thought to be expressed only in ciliated cells, were also found to be expressed in $\mathrm{CD}^{+}{ }^{\mathrm{T}}$ cells ${ }^{79,80}$. Several components of the IFT complex - IFT20, together with IFT57 and IFT52 - have been shown to maintain TCR signalling at the T cell synapse after contact with an APC, by regulating TCR recycling and recruitment of LAT to the synapse ${ }^{79-81}$. Furthermore, RAB8A/B which play a role in ciliogenesis ${ }^{82}$, have also been shown to contribute to vesicle-mediated TCR targeting to the immune synapse ${ }^{23,83}$. Intriguingly, ciliary TGF $\beta$ signalling has also been shown to be regulated by endocytosis at the ciliary pocket ${ }^{84}$. 
In addition to their roles in focusing signalling, both primary cilia and immune synapses form specialized $\mathrm{Ca}^{2+}$ signalling structures. A heteromeric transient receptor potential (TRP) channel, PKD1L1-PKD2L1, has been found to control local $\mathrm{Ca}^{2+}$ levels in the cilium; mice lacking PKD1L1-PKD2L1 show reduced Hedgehog $(\mathrm{Hh})$ signalling as assessed by GLI1 expression (see next section) ${ }^{85,86}$. Interestingly, $\mathrm{Ca}^{2+}$ influx is also generated locally at the synapse through the STIM1-activated $\mathrm{Ca}^{2+}$ release-activated $\mathrm{Ca}^{2+}$ (CRAC) channel ORAI1 in the plasma membrane and is required for effective TCR signalling 87.

Secretion at the immune synapse is well characterized; more recently, it has emerged that the primary cilium can shed vesicles. Primary cilia of neuroepithelial cells release membrane particles that are enriched for the membrane-organizing protein prominin-1 (also known as CD133) ${ }^{88}$, the cilia of neuronal cells shed vesicles containing G-protein-coupled receptors ${ }^{89}$, and the cilia of Caenorhabditis elegans sensory neurons release microvesicles that might be involved in communication and mating behaviour ${ }^{90}$. The flagellum of the unicellular alga Chlamydomonas also sheds membrane vesicles and these ciliary ectosomes carry proteolytic enzymes that digest the extracellular matrix to free daughter cells following mitosis ${ }^{91}$. Similarly, T cells secrete vesicles at the synapse: not only cytotoxic granules, but also microvesicles carrying bioactive CD95L (also known as FASL) and APO2L, microRNAs and TCRs ${ }^{92-94}$.

Hedgehog (Hh) signalling

Primary cilia have a role in many different signalling pathways, but the pathway that depends most on the primary cilium is $\mathrm{Hh}$ signalling ${ }^{95-98}$. First discovered in Drosophila ${ }^{99}$, the Hh pathway also has a key role in vertebrate embryonic development by regulating cell proliferation, differentiation and tissue patterning and adult tissue homeostasis ${ }^{100}$. Hh signalling is initiated when one of the three mammalian Hh ligands (Sonic $\mathrm{Hh}(\mathrm{SHH})$, Desert $\mathrm{Hh}$ $(\mathrm{DHH})$ or Indian $\mathrm{Hh}(\mathrm{IHH})$ ) binds to the transmembrane receptor patched $(\mathrm{PTCH})$ on a Hh-responsive cell. Ligand binding to $\mathrm{PTCH}$ allows the 
transmembrane protein smoothened (SMO) to translocate to the primary cilium, where it activates the glioma-associated oncogene (GLI) family of transcription factors, which translocate to the nucleus initiating the transcription of $\mathrm{Hh}$ target genes ${ }^{101}$ (Figure 6a).

In the immune system, pioneering work by the Crompton lab and others has uncovered roles for the Hh signalling pathway during $\mathrm{T}$ cell development in the thymus as well as during the differentiation of naive $\mathrm{CD}^{+}{ }^{+} \mathrm{T}$ cells into $\mathrm{T}$ helper $2\left(T_{H} 2\right)$ cells ${ }^{102,103}$. A role for Hh signalling at the synapse formed by $\mathrm{CD}^{+} \mathrm{T}$ cells has also emerged, with the discovery that TCR activation triggers not only the well-characterized downstream signalling pathways, but also gives rise to $\mathrm{Hh}$ signalling ${ }^{49}$ (Figure 6b). Experiments that inhibited the $\mathrm{Hh}$ signalling pathway in $\mathrm{CD}^{+} \mathrm{T}$ cells, either genetically (by conditional deletion of SMO) or by use of small molecule inhibitors, resulted in a loss of CTL-mediated cytotoxicity. Although TCR signalling and granule contents were unaffected in CTLs in which Hh signalling was selectively inhibited, these cells had a reduced clearance of actin from and centrosome polarization to the cytolytic synapse, leading to a loss of granule secretion. Further analysis revealed that $\mathrm{Hh}$ signalling is required for the upregulation of RAC1, a protein that has an important role in both actin and microtubule dynamics ${ }^{45,46}$. Thus, it appears that $\mathrm{Hh}$ signalling is required during the transition from a naïve $\mathrm{CD} 8^{+} \mathrm{T}$ cell to a $\mathrm{CTL}$, when it upregulates proteins required for actin reorganisation, centrosome polarisation and granule delivery (Figure 6c). Interestingly, proteins in the Hh signalling pathway may also have a direct role at the synapse. SMO has been shown to have a transcription-independent role in lamellipodia formation ${ }^{104}$ and to stimulate RAC1 and RHOA in SHH-induced fibroblast migration ${ }^{105}$, as well as to activate RAC1 via release of the RAC1-specific GEF TIAM1 in SHH-induced dendritic spine formation in neurons ${ }^{106}$. It is therefore possible that SMO might have a similar role in $\mathrm{T}$ cells.

Some interesting aspects of $\mathrm{Hh}$ signalling in $\mathrm{CD}^{+} \mathrm{T}$ cells distinguish this pathway from $\mathrm{Hh}$ signalling in $\mathrm{CD} 4^{+} \mathrm{T}$ cells and other systems. Conventional Hh signalling involves paracrine signalling between two different cells, with the 
Hh ligand secreted from one cell and signalling to another cell that expresses the $\mathrm{Hh}$ receptor ${ }^{101}$. However, in $\mathrm{CD}^{+} \mathrm{T}$ cells, Hh signalling seems to be intracellular. $\mathrm{CD}^{+} \mathrm{T}$ cells synthesise only one form of $\mathrm{Hh}$ ligand, $\mathrm{IHH}$, which is not processed for secretion and seems to bind to its receptor PTCH intracellularly on vesicles ${ }^{49}$. This unusual intracellular signalling pathway makes good biological sense, as $\mathrm{CD}^{+} \mathrm{T}$ cells would not be susceptible to unpredictable external $\mathrm{Hh}$ gradients when killing specific target cells throughout the body.

\section{Centrosome docking}

Exactly how centrosome docking at the synapse occurs is of interest for several reasons. There are some important differences between synapses and cilia. Cilia are stable structures that persist for days, whereas immune synapses are transient and in CTLs they exist for only a few minutes. In CTLs, therefore, it is important that stable contacts are not formed. Furthermore, several papers have challenged the idea that the centrosome contacts the plasma membrane directly in immune synapses and suggest that the centrosome simply polarizes towards the synapse with granules being delivered via short microtubules that bridge the gap between the centrosome and the site of secretion at the plasma membrane ${ }^{107,108 .}$

Centrosome docking during ciliogenesis is well understood, and many of the structures and proteins that are required for docking and cilia formation have been identified ${ }^{109}$. In brief, the centrosome is composed of two centrioles and surrounding pericentriolar material. The mature centriole is termed the mother, and can be distinguished by the presence of subdistal and distal appendages. During ciliogenesis, it is always the mother centriole (sometimes known as the basal body) that contacts the plasma membrane via its distal appendages. Several distal appendage proteins have been identified as being essential for docking of the mother centriole and subsequent ciliogenesis including CEP83 ${ }^{110}$. With this knowledge in hand, we asked whether centrosome docking at the immune synapse occurred via the distal appendages of the mother centriole and whether CEP83 was required. Using electron microscopy tomography to obtain $3 \mathrm{D}$ images of the centriole pair at 
the CTL synapse ${ }^{111}$, further similarities between immune synapses and cilia were identified. The mother centriole docked at the plasma membrane of the immune synapse via its distal appendages, with the microtubules radiating out from the subdistal appendages and providing a mechanism of granule delivery directly to the synapse membrane. CEP83 also seemed to be required for centrosome docking in CTLs, with knock-down of the protein inhibiting granule release in this study. One interesting complication was that because CTLs are rapidly proliferating cells, many contained multiple centriole pairs; although these polarized together during synapse formation, only one mother centriole seemed to dock at the membrane and organize the microtubules for cytolytic granule secretion.

If centrosome docking at the synapse so closely resembles that during ciliogenesis, then why do cilia not form at the synapse? Once again insights from studies of cilia have provided the answers. In order for a cilium to extend from the docked centrosome at the plasma membrane, the protein CP110 needs to dissociate from the end of the mother centriole ${ }^{112}$. However, CP110 remains associated with the docked mother centriole at the immune synapse and therefore ciliogenesis does not occur ${ }^{111}$. Thus the synapse mimics the early stages of ciliogenesis only and centrosome docking at the synapse is transient. Interestingly in serum-starved, transformed $B$ and $T$ cell lines in which CP110 is depleted, cilia formation can be made to occur in $10-15 \%$ of cells ${ }^{113}$. The retention of CP110 provides an important mechanism by which CTLs can engage the centrosome with the synapse only transiently and therefore undergo serial killing of multiple targets.

\section{Conclusions and perspectives}

The structural and functional similarities between immune synapses and cilia are intriguing. The first description of the parallels between synapses and cilia 5,71 resulted in the identification of novel molecular mechanisms that have a role at the immune synapse $49,79,80$. More broadly, these functional similarities raise the possibility that the adaptive immune system has modified an ancient organelle to create a sensory and signalling structure in order to focus secretion and provide a precise and readily regulated mechanism for 
communicating between cells. Using a cilium as a sensory organelle is no novelty. In Paramecium tetraurealis (a ciliated unicellular protozoa), secreted granules, called trichocysts, associate with microtubules emanating from the basal body of the cilium and show minus-end directed transport towards the point of secretion. Recent studies have shown that $\mathrm{Ca}^{2+}$ channels regulate both ciliary activity and exocytosis by not only the influx of extracellular $\mathrm{Ca}^{2+}$, but also the release of $\mathrm{Ca}^{2+}$ from specialized alveolar sacks, which is reminiscent of $\mathrm{Ca}^{2+}$ release from secretory lysosomes found in T cells ${ }^{114,115}$. Thus, this seems conceptually very similar to the secretion of cytolytic granules.

Although this Review has focused on CTLs, centrosome polarization also occurs in $\mathrm{CD}^{+}{ }^{\mathrm{T}}$ cells ${ }^{10}$, B cells ${ }^{116}$, NK cells, invariant NKT (iNKT) cells ${ }^{51}$ and dendritic cells ${ }^{117}$, making it likely that many of the mechanisms studied in CTLs and $\mathrm{CD}^{+} \mathrm{T}$ cells will be shared by other immune cells. It is important to note that cytolytic $\mathrm{CD}^{+} \mathrm{T}$ cells, capable of secreting perforin-containing granules ${ }^{118}$, have been identified as having roles during viral infections ${ }^{119}$ and cancer ${ }^{120}$, and with new insights about their development and differentiation now emerging ${ }^{121}$, much may be learned from these cells. What role ciliary pathways, such as $\mathrm{Hh}$ signalling, might have in non-T cell immune cells will be important to discover. The finding that Hh signalling has a crucial role in the CTL effector mechanism has major clinical implications. Aberrant $\mathrm{Hh}$ signalling has been associated with the development and progression of many different types of tumour ${ }^{122}$ and consequently $\mathrm{Hh}$ inhibitors are in clinical trials as treatment for various cancers ${ }^{123,124}$. Our observation that $\mathrm{Hh}$ signalling is important for CTL killing suggests that whatever beneficial effects $\mathrm{Hh}$ inhibitors might have, they will also diminish the immune response against the tumour.

Elucidating the role of $\mathrm{Hh}$ signalling in the peripheral immune system will be an important task in the future and might benefit not only cancer immunotherapy but also vaccination and the treatment of infection. 


\section{References}

1. Dustin, M.L. \& Springer, T.A. T-cell receptor cross-linking transiently stimulates adhesiveness through LFA-1. Nature 341, 619-24 (1989).

2. Potter, T.A., Grebe, K., Freiberg, B. \& Kupfer, A. Formation of supramolecular activation clusters on fresh ex vivo CD8+ T cells after engagement of the $T$ cell antigen receptor and CD8 by antigenpresenting cells. Proc Natl Acad Sci U S A 98, 12624-9 (2001).

3. Monks, C.R., Freiberg, B.A., Kupfer, H., Sciaky, N. \& Kupfer, A. Threedimensional segregation of supramolecular activation clusters in $\mathrm{T}$ cells. Nature 395, 82-6 (1998).

4. O'Keefe, J.P., Blaine, K., Alegre, M.L. \& Gajewski, T.F. Formation of a central supramolecular activation cluster is not required for activation of naive CD8+ T cells. Proc Natl Acad Sci U S A 101, 9351-6 (2004).

5. Stinchcombe, J.C., Majorovits, E., Bossi, G., Fuller, S. \& Griffiths, G.M. Centrosome polarization delivers secretory granules to the immunological synapse. Nature 443, 462-5 (2006).

6. Sanderson, C.J. \& Glauert, A.M. The mechanism of T cell mediated cytotoxicity. V. Morphological studies by electron microscopy. Proc $R$ Soc Lond B Biol Sci 198, 315-323 (1977).

7. Sanderson, C.J. \& Glauert, A.M. The mechanism of T-cell mediated cytotoxicity. VI. T-cell projections and their role in target cell killing. Immunology 36, 119-29 (1979).

An early description of the role of membrane projections in the initial contact between a killer cell and target cell.

8. Geiger, B., Rosen, D. \& Berke, G. Spatial relationships of microtubuleorganizing centers and the contact area of cytotoxic $\mathrm{T}$ lymphocytes and target cells. J Cell Biol 95, 137-43 (1982).

9. Choudhuri, K., Wiseman, D., Brown, M.H., Gould, K. \& van der Merwe, P.A. T-cell receptor triggering is critically dependent on the dimensions of its peptide-MHC ligand. Nature 436, 578-82 (2005).

10. Ueda, H., Morphew, M.K., Mclntosh, J.R. \& Davis, M.M. CD4+ T-cell synapses involve multiple distinct stages. Proc Natl Acad Sci U S A 108, 17099-104 (2011).

11. Jenkins, M.R. et al. Distinct structural and catalytic roles for Zap70 in formation of the immunological synapse in CTL. Elife 3, e01310 (2014).

12. Balagopalan, L., Sherman, E., Barr, V.A. \& Samelson, L.E. Imaging techniques for assaying lymphocyte activation in action. Nat Rev Immunol 11, 21-33 (2011).

13. Grakoui, A. et al. The immunological synapse: a molecular machine controlling T cell activation. Science 285, 221-7 (1999).

14. Varma, R., Campi, G., Yokosuka, T., Saito, T. \& Dustin, M.L. T cell receptor-proximal signals are sustained in peripheral microclusters and terminated in the central supramolecular activation cluster. Immunity 25, 117-27 (2006).

15. Yi, J., Wu, X.S., Crites, T. \& Hammer, J.A., 3rd. Actin retrograde flow and actomyosin II arc contraction drive receptor cluster dynamics at the immunological synapse in Jurkat T cells. Mol Biol Cell 23, 834-52 (2012). 
16. Beemiller, P., Jacobelli, J. \& Krummel, M.F. Integration of the movement of signaling microclusters with cellular motility in immunological synapses. Nat Immunol 13, 787-95 (2012).

17. Hashimoto-Tane, A. et al. Dynein-driven transport of $T$ cell receptor microclusters regulates immune synapse formation and $T$ cell activation. Immunity 34, 919-31 (2011).

18. Martin-Cofreces, N.B. et al. End-binding protein 1 controls signal propagation from the T cell receptor. EMBO J 31, 4140-52 (2012).

19. Das, V. et al. Activation-induced polarized recycling targets $\mathbf{T}$ cell antigen receptors to the immunological synapse; involvement of SNARE complexes. Immunity 20, 577-88 (2004).

Together with reference 23 , this paper describes the role of vesicle fusion in modulating TCR signalling.

20. Purbhoo, M.A. et al. Dynamics of subsynaptic vesicles and surface microclusters at the immunological synapse. Sci Signal 3, ra36 (2010).

21. Williamson, D.J. et al. Pre-existing clusters of the adaptor Lat do not participate in early T cell signaling events. Nat Immunol 12, 655-62 (2011).

22. Larghi, P. et al. VAMP7 controls T cell activation by regulating the recruitment and phosphorylation of vesicular Lat at TCR-activation sites. Nat Immunol 14, 723-31 (2013).

23. Soares, H. et al. Regulated vesicle fusion generates signaling nanoterritories that control $\mathrm{T}$ cell activation at the immunological synapse. J Exp Med 210, 2415-33 (2013).

Together with reference 19, this paper describes the role of vesicle fusion in modulating TCR signalling.

24. Ritter, A.T. et al. Actin depletion initiates events leading to granule secretion at the immunological synapse. Immunity 42, 864-76 (2015).

This study reports 4D imaging of the steps leading to secretion at the cytolytic synapse of a CTL.

25. Raucher, D. et al. Phosphatidylinositol 4,5-bisphosphate functions as a second messenger that regulates cytoskeleton-plasma membrane adhesion. Cell 100, 221-8 (2000).

26. Janmey, P.A. \& Lindberg, U. Cytoskeletal regulation: rich in lipids. Nat Rev Mol Cell Biol 5, 658-66 (2004).

27. Comrie, W.A., Babich, A. \& Burkhardt, J.K. F-actin flow drives affinity maturation and spatial organization of LFA-1 at the immunological synapse. J Cell Biol 208, 475-91 (2015).

This study describes an important role for actin flow in activating integrins at the immune synapse.

28. Wei, S.Y. et al. Protein kinase C-delta and -beta coordinate flowinduced directionality and deformation of migratory human blood Tlymphocytes. J Mol Cell Biol 6, 458-72 (2014).

29. Kumari, S. et al. Actin foci facilitate activation of the phospholipase C-gamma in primary $\mathrm{T}$ lymphocytes via the WASP pathway. eLife 4; 04953 (2015).

This study reports a possible role for actin-rich protrusions at the $\mathrm{T}$ cell synapse. 
30. Tsun, A. et al. Centrosome docking at the immunological synapse is controlled by Lck signaling. J Cell Biol 192, 663-74 (2011).

31. Lowin-Kropf, B., Shapiro, V.S. \& Weiss, A. Cytoskeletal polarization of $T$ cells is regulated by an immunoreceptor tyrosine-based activation motif-dependent mechanism. J Cell Biol 140, 861-71 (1998).

32. Kuhne, M.R. et al. Linker for activation of T cells, zeta-associated protein-70, and Src homology 2 domain-containing leukocyte protein76 are required for TCR-induced microtubule-organizing center polarization. J Immunol 171, 860-6 (2003).

33. Spitaler, M., Emslie, E., Wood, C.D. \& Cantrell, D. Diacylglycerol and protein kinase $\mathrm{D}$ localization during $\mathrm{T}$ lymphocyte activation. Immunity 24, 535-46 (2006).

34. Kupfer, A., Swain, S.L. \& Singer, S.J. The specific direct interaction of helper T cells and antigen-presenting B cells. II. Reorientation of the microtubule organizing center and reorganization of the membraneassociated cytoskeleton inside the bound helper T cells. J Exp Med 165, 1565-80 (1987).

35. Yi, J. et al. Centrosome repositioning in $\mathrm{T}$ cells is biphasic and driven by microtubule end-on capture-shrinkage. J Cell Biol 202, 779-92 (2013).

This paper provides exceptional mechanistic insights into centrosome polarization via pioneer microtubules.

36. Quann, E.J., Merino, E., Furuta, T. \& Huse, M. Localized diacylglycerol drives the polarization of the microtubule-organizing center in T cells. Nat Immunol 10, 627-35 (2009).

37. Liu, X., Kapoor, T.M., Chen, J.K. \& Huse, M. Diacylglycerol promotes centrosome polarization in $\mathrm{T}$ cells via reciprocal localization of dynein and myosin II. Proc Natl Acad Sci U S A 110, 11976-81 (2013).

Together with reference 42 , this study describes a role for dynein in centrosome polarization to the synapse.

38. Quann, E.J., Liu, X., Altan-Bonnet, G. \& Huse, M. A cascade of protein kinase $\mathrm{C}$ isozymes promotes cytoskeletal polarization in T cells. Nat Immunol 12, 647-54 (2011).

39. Bertrand, F. et al. Activation of the ancestral polarity regulator protein kinase $\mathrm{C}$ zeta at the immunological synapse drives polarization of $\mathrm{Th}$ cell secretory machinery toward APCs. J Immunol 185, 2887-94 (2010).

40. Ludford-Menting, M.J. et al. A network of PDZ-containing proteins regulates $T$ cell polarity and morphology during migration and immunological synapse formation. Immunity 22, 737-48 (2005).

41. Lin, J., Hou, K.K., Piwnica-Worms, H. \& Shaw, A.S. The polarity protein Par1b/EMK/MARK2 regulates T cell receptor-induced microtubuleorganizing center polarization. J Immunol 183, 1215-21 (2009).

42. Combs, J. et al. Recruitment of dynein to the Jurkat immunological synapse. Proc Natl Acad Sci U S A 103, 14883-8 (2006).

Together with reference 37 , this study describes a role for dynein in centrosome polarization to the synapse. 
43. Martin-Cofreces, N.B. et al. MTOC translocation modulates IS formation and controls sustained T cell signaling. J Cell Biol 182, 95162 (2008).

44. Kuhn, J.R. \& Poenie, M. Dynamic polarization of the microtubule cytoskeleton during CTL-mediated killing. Immunity 16, 111-21 (2002).

45. Wittmann, T., Bokoch, G.M. \& Waterman-Storer, C.M. Regulation of leading edge microtubule and actin dynamics downstream of Rac1. J Cell Biol 161, 845-51 (2003).

46. Wittmann, T., Bokoch, G.M. \& Waterman-Storer, C.M. Regulation of microtubule destabilizing activity of Op18/stathmin downstream of Rac1. J Biol Chem 279, 6196-203 (2004).

47. Gomez, T.S. et al. Formins regulate the actin-related protein $2 / 3$ complex-independent polarization of the centrosome to the immunological synapse. Immunity 26, 177-90 (2007).

48. Filbert, E.L., Le Borgne, M., Lin, J., Heuser, J.E. \& Shaw, A.S. Stathmin regulates microtubule dynamics and microtubule organizing center polarization in activated T cells. J Immunol 188, 5421-7 (2012).

49. de la Roche, $M$. et al. Hedgehog signaling controls $\mathbf{T}$ cell killing at the immunological synapse. Science 342, 1247-50 (2013).

This paper describes a role for ciliary Hh signalling in CTL secretion.

50. Zyss, D., Ebrahimi, H. \& Gergely, F. Casein kinase I delta controls centrosome positioning during T cell activation. J Cell Biol 195, 781-97 (2011).

The first description of a centrosomal protein that is required for centrosome polarization in T cells.

51. Stinchcombe, J.C. et al. Centriole polarisation to the immunological synapse directs secretion from cytolytic cells of both the innate and adaptive immune systems. BMC Biol 9, 45 (2011).

52. Bryceson, Y.T., March, M.E., Barber, D.F., Ljunggren, H.G. \& Long, E.O. Cytolytic granule polarization and degranulation controlled by different receptors in resting NK cells. J Exp Med 202, 1001-12 (2005).

53. March, M.E. \& Long, E.O. beta2 integrin induces TCRzeta-Sykphospholipase C-gamma phosphorylation and paxillin-dependent granule polarization in human NK cells. J Immunol 186, 2998-3005 (2011).

54. Barber, D.F., Faure, M. \& Long, E.O. LFA-1 contributes an early signal for NK cell cytotoxicity. J Immunol 173, 3653-9 (2004).

55. Au-Yeung, B.B. et al. A genetically selective inhibitor demonstrates a function for the kinase Zap70 in regulatory $T$ cells independent of its catalytic activity. Nat Immunol 11, 1085-92 (2010).

56. Zhang, M., March, M.E., Lane, W.S. \& Long, E.O. A signaling network stimulated by beta2 integrin promotes the polarization of lytic granules in cytotoxic cells. Sci Signal 7, ra96 (2014). This study uses a mass spectrometry approach to identify the signalling network initiated by $\beta_{2}$ integrin signalling that is required for granule and centrosome polarization towards the synapse in NK cells.

57. Legate, K.R., Montanez, E., Kudlacek, O. \& Fassler, R. ILK, PINCH and parvin: the tIPP of integrin signalling. Nat Rev Mol Cell Biol 7, 2031 (2006). 
58. Robertson, L.K. \& Ostergaard, H.L. Paxillin associates with the microtubule cytoskeleton and the immunological synapse of CTL through its leucine-aspartic acid domains and contributes to microtubule organizing center reorientation. J Immunol 187, 5824-33 (2011).

59. Gismondi, A. et al. Cutting edge: functional role for proline-rich tyrosine kinase 2 in NK cell-mediated natural cytotoxicity. J Immunol 164, 22726 (2000).

60. Sancho, D. et al. The tyrosine kinase PYK-2/RAFTK regulates natural killer (NK) cell cytotoxic response, and is translocated and activated upon specific target cell recognition and killing. J Cell Biol 149, 1249-62 (2000).

61. Zhang, Q. et al. Combined immunodeficiency associated with DOCK8 mutations. N Engl J Med 361, 2046-55 (2009).

62. Mizesko, M.C. et al. Defective actin accumulation impairs human natural killer cell function in patients with dedicator of cytokinesis 8 deficiency. J Allergy Clin Immunol 131, 840-8 (2013).

63. Ham, H. et al. Dedicator of cytokinesis 8 interacts with talin and Wiskott-Aldrich syndrome protein to regulate NK cell cytotoxicity. $J$ Immunol 190, 3661-9 (2013).

64. Ham, H., Huynh, W., Schoon, R.A., Vale, R.D. \& Billadeau, D.D. HkRP3 is a microtubule-binding protein regulating lytic granule clustering and NK cell killing. J Immunol 194, 3984-96 (2015).

65. Pieters, J., Muller, P. \& Jayachandran, R. On guard: coronin proteins in innate and adaptive immunity. Nat Rev Immunol 13, 510-8 (2013).

66. Mace, E.M. \& Orange, J.S. Lytic immune synapse function requires filamentous actin deconstruction by Coronin 1A. Proc Natl Acad Sci U S A 111, 6708-6713 (2014).

Patients lacking coronin 1A show defective NK cell-mediated killing.

67. Rak, G.D., Mace, E.M., Banerjee, P.P., Svitkina, T. \& Orange, J.S. Natural killer cell lytic granule secretion occurs through a pervasive actin network at the immune synapse. PLoS Biol 9, e1001151 (2011).

68. Brown, A.C. et al. Remodelling of cortical actin where lytic granules dock at natural killer cell immune synapses revealed by superresolution microscopy. PLoS Biol 9, e1001152 (2011).

69. Wheatley, D.N. Primary cilia in normal and pathological tissues. Pathobiology 63, 222-38 (1995).

70. Hildebrandt, F. \& Otto, E. Cilia and centrosomes: a unifying pathogenic concept for cystic kidney disease? Nat Rev Genet 6, 928-40 (2005).

71. Stinchcombe, J.C. \& Griffiths, G.M. Secretory mechanisms in cellmediated cytotoxicity. Annu Rev Cell Dev Biol 23, 495-517 (2007).

72. Griffiths, G.M., Tsun, A. \& Stinchcombe, J.C. The immunological synapse: a focal point for endocytosis and exocytosis. J Cell Biol 189, 399-406 (2010).

73. Singla, V. \& Reiter, J.F. The primary cilium as the cell's antenna: signaling at a sensory organelle. Science 313, 629-33 (2006).

74. Francis, S.S., Sfakianos, J., Lo, B. \& Mellman, I. A hierarchy of signals regulates entry of membrane proteins into the ciliary membrane domain in epithelial cells. J Cell Biol 193, 219-33 (2011). 
75. Jensen, V.L. et al. Formation of the transition zone by Mks5/Rpgrip1L establishes a ciliary zone of exclusion (CIZE) that compartmentalises ciliary signalling proteins and controls PIP2 ciliary abundance. EMBO J 34, 2537-56 (2015).

76. Garcia-Gonzalo, F.R. et al. Phosphoinositides Regulate Ciliary Protein Trafficking to Modulate Hedgehog Signaling. Dev Cell 34, 400-9 (2015).

77. Chavez, M. et al. Modulation of Ciliary Phosphoinositide Content Regulates Trafficking and Sonic Hedgehog Signaling Output. Dev Cell 34, 338-50 (2015).

78. Rosenbaum, J.L. \& Witman, G.B. Intraflagellar transport. Nat Rev Mol Cell Biol 3, 813-25 (2002).

79. Finetti, F. et al. Specific recycling receptors are targeted to the immune synapse by the intraflagellar transport system. J Cell Sci 127, 1924-37 (2014).

80. Finetti, F. et al. Intraflagellar transport is required for polarized recycling of the TCR/CD3 complex to the immune synapse. Nat Cell Biol 11, 1332-9 (2009).

81. Vivar, O.I. et al. IFT20 controls LAT recruitment to the immune synapse and T-cell activation in vivo. Proc Natl Acad Sci U S A 113, 386-91 (2016).

82. Sato, T. et al. Rab8a and Rab8b are essential for several apical transport pathways but insufficient for ciliogenesis. J Cell Sci 127, 42231 (2014).

83. Finetti, F. et al. The small GTPase Rab8 interacts with VAMP-3 to regulate the delivery of recycling T-cell receptors to the immune synapse. J Cell Sci 128, 2541-52 (2015).

84. Clement, C.A. et al. TGF-beta signaling is associated with endocytosis at the pocket region of the primary cilium. Cell Rep 3, 1806-14 (2013).

85. Delling, M., DeCaen, P.G., Doerner, J.F., Febvay, S. \& Clapham, D.E. Primary cilia are specialized calcium signalling organelles. Nature 504, 311-4 (2013).

86. DeCaen, P.G., Delling, M., Vien, T.N. \& Clapham, D.E. Direct recording and molecular identification of the calcium channel of primary cilia. Nature 504, 315-8 (2013).

87. Hogan, P.G., Lewis, R.S. \& Rao, A. Molecular basis of calcium signaling in lymphocytes: STIM and ORAI. Annu Rev Immunol 28, 491533 (2010).

88. Dubreuil, V., Marzesco, A.M., Corbeil, D., Huttner, W.B. \& WilschBrauninger, M. Midbody and primary cilium of neural progenitors release extracellular membrane particles enriched in the stem cell marker prominin-1. J Cell Biol 176, 483-95 (2007).

89. Soetedjo, L. \& Jin, H. Agonist-Induced GPCR Shedding from the Ciliary Surface Is Dependent on ESCRT-III and VPS4. Curr Biol 24, 509-18 (2014).

90. Wang, J. et al. C. elegans Ciliated Sensory Neurons Release Extracellular Vesicles that Function in Animal Communication. Curr Biol 24, 519-25 (2014).

91. Wood, C.R., Huang, K., Diener, D.R. \& Rosenbaum, J.L. The cilium secretes bioactive ectosomes. Curr Biol 23, 906-11 (2013). 
92. Martinez-Lorenzo, M.J. et al. Activated human T cells release bioactive Fas ligand and APO2 ligand in microvesicles. J Immunol 163, 1274-81 (1999).

93. Mittelbrunn, M. et al. Unidirectional transfer of microRNA-loaded exosomes from T cells to antigen-presenting cells. Nat Commun 2, 282 (2011).

94. Choudhuri, K. et al. Polarized release of T-cell-receptor-enriched microvesicles at the immunological synapse. Nature 507, 118-23 (2014).

95. Huangfu, D. et al. Hedgehog signalling in the mouse requires intraflagellar transport proteins. Nature 426, 83-7 (2003).

96. Corbit, K.C. et al. Vertebrate Smoothened functions at the primary cilium. Nature 437, 1018-21 (2005).

97. Haycraft, C.J. et al. Gli2 and Gli3 localize to cilia and require the intraflagellar transport protein polaris for processing and function. PLOS Genet 1, e53 (2005).

98. Rohatgi, R., Milenkovic, L. \& Scott, M.P. Patched1 regulates hedgehog signaling at the primary cilium. Science 317, 372-6 (2007).

99. Nusslein-Volhard, C. \& Wieschaus, E. Mutations affecting segment number and polarity in Drosophila. Nature 287, 795-801 (1980).

100. Goetz, S.C. \& Anderson, K.V. The primary cilium: a signalling centre during vertebrate development. Nat Rev Genet 11, 331-44 (2010).

101. Briscoe, J. \& Therond, P.P. The mechanisms of Hedgehog signalling and its roles in development and disease. Nat Rev Mol Cell Biol 14, 416-29 (2013).

102. Furmanski, A.L. et al. Tissue-derived hedgehog proteins modulate Th differentiation and disease. J Immunol 190, 2641-9 (2013)

103. Crompton, T., Outram, S.V. \& Hager-Theodorides, A.L. Sonic hedgehog signalling in T-cell development and activation. Nat Rev Immunol 7, 726-35 (2007).

104. Bijlsma, M.F., Borensztajn, K.S., Roelink, H., Peppelenbosch, M.P. \& Spek, C.A. Sonic hedgehog induces transcription-independent cytoskeletal rearrangement and migration regulated by arachidonate metabolites. Cell Signal 19, 2596-604 (2007).

105. Polizio, A.H. et al. Heterotrimeric Gi proteins link Hedgehog signaling to activation of Rho small GTPases to promote fibroblast migration. J Biol Chem 286, 19589-96 (2011).

106. Sasaki, N., Kurisu, J. \& Kengaku, M. Sonic hedgehog signaling regulates actin cytoskeleton via Tiam1-Rac1 cascade during spine formation. Mol Cell Neurosci 45, 335-44 (2010).

107. Kurowska, M. et al. Terminal transport of lytic granules to the immune synapse is mediated by the kinesin-1/SIp3/Rab27a complex. Blood 119, 3879-89 (2012).

108. Bertrand, F. et al. An initial and rapid step of lytic granule secretion precedes microtubule organizing center polarization at the cytotoxic $T$ lymphocyte/target cell synapse. Proc Natl Acad Sci U S A 110, 6073-8 (2013).

109. Wei, Q., Ling, K. \& Hu, J. The essential roles of transition fibers in the context of cilia. Curr Opin Cell Biol 35, 98-105 (2015). 
110. Tanos, B.E. et al. Centriole distal appendages promote membrane docking, leading to cilia initiation. Genes Dev 27, 163-8 (2013). An important paper in revealing the role of CEP83 in centrosome docking.

111. Stinchcombe, J.C. et al. Mother Centriole Distal Appendages Mediate Centrosome Docking at the Immunological Synapse and Reveal Mechanistic Parallels with Ciliogenesis. Curr Biol 25, 323944 (2015).

This study shows that the centrosome docks directly at the synapse via distal appendages of the mother centriole and that CEP83 depletion reduces killing by $C T L s$.

112. Spektor, A., Tsang, W.Y., Khoo, D. \& Dynlacht, B.D. Cep97 and CP110 suppress a cilia assembly program. Cell 130, 678-90 (2007).

113. Prosser, S.L. \& Morrison, C.G. Centrin2 regulates CP110 removal in primary cilium formation. J Cell Biol 208, 693-701 (2015).

Cilia can be forced to form in $B$ and $T$ cells, showing the machinery is present but not used.

114. Plattner, H. Calcium signalling in the ciliated protozoan model, Paramecium: strict signal localisation by epigenetically controlled positioning of different $\mathrm{Ca}(2)(+)$-channels. Cell Calcium 57, 203-13 (2015).

115. Davis, L.C. et al. NAADP activates two-pore channels on T cell cytolytic granules to stimulate exocytosis and killing. Curr Biol 22, 2331-7 (2012).

116. Yuseff, M.I. et al. Polarized secretion of lysosomes at the B cell synapse couples antigen extraction to processing and presentation. Immunity 35, 361-74 (2011).

117. Pulecio, J. et al. Cdc42-mediated MTOC polarization in dendritic cells controls targeted delivery of cytokines at the immune synapse. $J$ Exp Med 207, 2719-32 (2010).

118. Beal, A.M. et al. Kinetics of early T cell receptor signaling regulate the pathway of lytic granule delivery to the secretory domain. Immunity 31, 632-42 (2009).

119. Jellison, E.R., Kim, S.K. \& Welsh, R.M. Cutting edge: MHC class IIrestricted killing in vivo during viral infection. J Immunol 174, 614-8 (2005).

120. Quezada, S.A. et al. Tumor-reactive CD4(+) T cells develop cytotoxic activity and eradicate large established melanoma after transfer into lymphopenic hosts. J Exp Med 207, 637-50 (2010).

121. Takeuchi, A. et al. CRTAM determines the CD4+ cytotoxic T lymphocyte lineage. J Exp Med 213, 123-38 (2016).

This study reveals the lineage regulation of cytolytic $\mathrm{CD} 4^{+} \mathrm{T}$ cells.

122. Scales, S.J. \& de Sauvage, F.J. Mechanisms of Hedgehog pathway activation in cancer and implications for therapy. Trends Pharmacol Sci 30, 303-12 (2009).

123. Ng, J.M. \& Curran, T. The Hedgehog's tale: developing strategies for targeting cancer. Nat Rev Cancer 11, 493-501 (2011).

124. Low, J.A. \& de Sauvage, F.J. Clinical experience with Hedgehog pathway inhibitors. J Clin Oncol 28, 5321-6 (2010). 
125. Sorokin, S.P. Reconstructions of centriole formation and ciliogenesis in mammalian lungs. J Cell Sci 3, 207-230 (1968).

126. Fisch, C. \& Dupuis-Williams, P. Ultrastructure of cilia and flagella back to the future! Biol Cell 103, 249-70 (2011).

127. Stinchcombe, J.C., Bossi, G., Booth, S. \& Griffiths, G.M. The immunological synapse of CTL contains a secretory domain and membrane bridges. Immunity 15, 751-61 (2001).

128. Poole, C.A. et al. Confocal analysis of primary cilia structure and colocalization with the Golgi apparatus in chondrocytes and aortic smooth muscle cells. Cell Biol Int 21, 483-94 (1997).

129. Kupfer, A., Dennert, G. \& Singer, S.J. The reorientation of the Golgi apparatus and the microtubule-organizing center in the cytotoxic effector cell is a prerequisite in the lysis of bound target cells. $\mathrm{J} \mathrm{Mol}$ Cell Immunol 2, 37-49 (1985).

130. Keady, B.T. et al. IFT25 links the signal-dependent movement of Hedgehog components to intraflagellar transport. Dev Cell 22, 940-51 (2012).

131. Nachury, M.V. How do cilia organize signalling cascades? Philos Trans $R$ Soc Lond B Biol Sci 369, 20130465 (2014).

132. Wheatley, D.N. Cilia and centrioles of the rat adrenal cortex. J Anat 101, 223-37 (1967).

\section{Acknowledgements}

GMG is funded by Wellcome Trust Principal Research Fellowship [103930],

MDR is now funded by a Sir Henry Dale Fellowship jointly funded by the Wellcome Trust and the Royal Society [107609]. 
Box 1: Structural and functional similarities between the immune synapse and the primary cilium

- The centrosome is docked at the cell surface membrane via distal appendages of the mother centriole in the primary cilium ${ }^{125,126}$ and the immune synapse ${ }^{111}$

- Cortical actin is reduced in the region of the primary cilium ${ }^{74}$ and the synapse ${ }^{127}$

- The Golgi apparatus and endocytic recycling compartments are polarized to the primary cilium ${ }^{128}$ and the immune synapse ${ }^{8,72,129}$

- The Hedgehog signalling pathway operates at the primary cilium ${ }^{95-98}$ and the immune synapse ${ }^{49}$

- Signalling component trafficking involves intraflagellar transport proteins in the primary cilium ${ }^{130,131}$ and the immune synapse ${ }^{79-81}$

- Localized membrane $\mathrm{Ca}^{2+}$ channels are present in the primary cilium 85,86 and the immune synapse ${ }^{87,115}$

Figure 1: Actin-rich interdigitations characterize the early but not the mature cytolytic synapse

a | Early synapse formation. Initial contact between the cytotoxic $T$ lymphocyte (CTL) and the target cell occurs via actin-rich filopodia that form an interdigitated contact site with the target cell, as seen in electron microscopy images, corresponding to an accumulation of actin across the synapse as seen by immunofluorescence microscopy. Shown in cartoon format are the initial contacts with TCR-peptide-MHC interactions at the tips of the actin-rich interdigitations. En face, these could appear as small clusters of $\mathrm{T}$ cell receptor (TCR) within an actin-rich synapse when viewed en face. At this early stage of synapse formation, the centrosome is not polarised and vesicles and secretory granules are dispersed within the cell. b | Mature synapse formation. Upon TCR engagement, the actin-rich lamellipodia focus at the periphery of the synapse, and electron microscopy shows extended areas of close flattened membrane contacts between CTL and target, with a secretory cleft (SC) in the centre. TCR microclusters coalesce at the centre of the synapse forming the cSMAC; the centrosome docks at the plasma membrane, and both cytotoxic granules and vesicles with TCR-signalling proteins move along microtubules towards the immune synapse.

The electron micrographs are reproduced with permission from ${ }^{11}$.

NL: nucleus, SG: secretory/cytotoxic granule, SC: secretory cleft, arrowhead: centriole 
Figure 2: The order of events leading to polarized secretion at the CTL synapse

Cytotoxic T lymphocytes (CTLs) are highly mobile cells that migrate through tissue in search of their cognate target cell. The leading edge is characterized by actin-rich lamellipodia while the centrosome (microtubule-organizing centre, MTOC) is localised in the uropod (a). When the CTL makes contact with a specific target cell (b), the actin (shown in red) from the initially actinrich CTL interface immediately starts to clear towards the periphery of the synapse (c). Then, membrane-associated T cell receptors (TCRs) cluster in the centre of the actin-depleted area forming the central supramolecular activation cluster (cSMAC), and the centrosome starts to move towards the establishing synapse (d). After $\sim 6 \mathrm{~min}$, the centrosome docks at the plasma membrane of the synapse where TCR signalling is occurring (e). The docked centrosome marks the point at which lytic granules containing cytotoxic perforin and granzymes are secreted and consequently kill the infected or tumour target cell $(\mathbf{f})$. Images from Y.A.

\section{Figure 3: Actin dynamics in migrating and synapsing CTL}

Cartoon depicting the rearward flow of actin (arrows) observed in migrating cytotoxic T lymphocytes (CTLs) (a), and CTLs synapsing with target cells (b). Immunofluorescent images showing both actin (red) and the actin-recruiting phosphoinositide, PIP2 (green), are reduced across the synapse compared to a control farnesyl membrane marker (green) (c) indicating a change in membrane composition and cortical actin recruitment. Images reproduced with permission from ${ }^{24}$.

\section{Figure 4: Regulation of centrosome polarization to the immune synapse}

Centrosome polarisation to the immune synapse is initiated when the $T$ cell receptor (TCR) binds cognate peptide-MHC complexes presented by an antigen-presenting cell (APC). The tyrosine kinase LCK is recruited to the TCR complex and phosphorylates ZAP70 ( $\zeta$-chain associated protein kinase of 70 $\mathrm{kDa}$ ). Activated ZAP70 in turn phosphorylates the adaptor protein linker for activation of $\mathrm{T}$ cells (LAT) and the LAT signalosome forms, including phospholipase CY1 (PLCY1) and SLP76 (SH2 domain-containing leukocyte protein of $76 \mathrm{kDa}$ ). PLCy1 converts Ptdlns $(4,5) \mathrm{P}_{2}$ (phosphatidylinositol-4,5bisphosphate) into DAG (diacylglycerol) and $\operatorname{lnsP}_{3}$ (inositol-1,4,5trisphosphate). DAG accumulates at the immune synapse, which leads to the recruitment of novel PKCs including PKC $\theta$. PKC $\theta$ inhibits nonmuscle myosin II (NMII) (a molecular motor protein that binds filamentous actin) and leads to the 
localization of NMII behind the centrosome at the rear of the cell (providing a 'pushing' force on the centrosome). PKC $\theta$ also promotes the accumulation of dynein (DYN) at the synapse. DYN is a minus-end-directed microtubule motor protein that reels the centrosome in by an end-on capture-shrinkage mechanism, thereby pulling the centrosome towards the synapse (see inset box). TCR stimulation also induces Hedgehog $(\mathrm{Hh})$ signalling, which leads to the expression of a $\mathrm{Hh}$-specific transcriptional target gene programme. The small Rho GTPase RAC1 is a Hh target gene that regulates pioneer microtubules and centrosome polarization. Pioneer microtubules and centrosome polarization are also regulated by stathmin, and the formins FMNL1, INF2 and DIA1. The role of microtubule dynamics is underscored by the role of the microtubule-stabilizing protein $\mathrm{CK} 1 \delta$ in centrosome polarization. $\mathrm{PKC} \zeta$, a component of the cell polarity-determining partitioning defective (PAR) complex, and the PAR family member PAR1B become activated by the TCR and are also implicated in centrosome polarization.

\section{Figure 5: Structural similarities between the cytolytic synapse and the primary cilium}

The cytolytic synapse, which forms when a cytotoxic $T$ lymphocyte (CTL) recognises specific antigen presented by $\mathrm{MHC}$ class I molecules on a target cell (a), bears striking morphological similarities with sites where a primary cilium is formed in ciliated cell types (b). In both structures, the mother centriole of the centrosome is 'docked' at the plasma membrane via distal appendage proteins (black arrows) and marks an area of focussed endo- and exocytosis with a polarized Golgi apparatus $(G)$ and endocytic recycling compartment, as well as secretory granules (SG; cytotoxic granules in CTLs). (The plasma membrane composition at the cytolytic synapse and the primary cilium (red) is different from the rest of the cell.) The electron micrographs are reproduced with permission from ${ }^{111}(a)$ and ${ }^{132}(b)$.

\section{Figure 6: Hedgehog signalling at the primary cilium and in $\mathrm{CD}^{+} \mathrm{T}$ cells}

a | In vertebrates, canonical Hedgehog $(\mathrm{Hh})$ signalling is associated with the primary cilium. The receptor Patched (PTCH) is localized at the base of the cilium and binds exogenous Hh ligands (Sonic $\mathrm{Hh}(\mathrm{SHH})$, Indian $\mathrm{Hh}(\mathrm{IHH})$ or Desert $\mathrm{Hh}(\mathrm{DHH})$ ) secreted by another Hh ligand-producing cell type. Upon ligand binding, PTCH releases its inhibition of the key signal transducer Smoothened (SMO) (through a mechanism that is poorly understood) and SMO moves into the cilium and becomes fully activated. This results in the dissociation of GLI transcription factors from SUFU in the cilium and the translocation of full-length, activated GLI transcription factors into the nucleus, 
where they initiate a Hh-specific transcriptional programme. (b) Cells of the hematopoietic lineage are thought to be unable to form primary cilia, but the immune synapse of $\mathrm{T}$ cells has marked structural similarities with sites of primary cilia formation, and naïve $\mathrm{CD} 8^{+} \mathrm{T}$ cells activate $\mathrm{Hh}$ signalling upon $\mathrm{T}$ cell receptor (TCR) stimulation. Our knowledge of $\mathrm{Hh}$ signalling in $\mathrm{CD}^{+} \mathrm{T}$ cells is rudimentary but the pathway seems to be unique compared with $\mathrm{Hh}$ signalling in primary cilia. Hh signalling in $\mathrm{CD}^{+} \mathrm{T}$ cells occurs solely intracellulary: $\mathrm{CD}^{+} \mathrm{T}$ cells produce $\mathrm{IHH}$ (not $\mathrm{SHH}$ and $\mathrm{DHH}$ ) themselves and the ligand colocalizes with the receptor PTCH on intracellular vesicles. SMO localizes to the synapse and GLI transcription factors initiate target gene transcription. (c) Upon TCR signalling, naïve $\mathrm{CD}^{+} \mathrm{T}$ cells not only proliferate massively and acquire cytotoxic T lymphocyte (CTL) effector functions, they also induce $\mathrm{Hh}$ signalling and upregulate expression of RAC1, a novel $\mathrm{Hh}$ target gene in $\mathrm{CD}^{+} \mathrm{T}$ cells that is required for the actin reorganization and centrosome polarization that is essential for effective target cell killing. Upon target cell recognition, CTLs not only refill their cytotoxic machinery but also boost $\mathrm{Hh}$ signalling to maintain high RAC1 levels. Fig. $6 c$ is modified with permission from ${ }^{49}$. 


\section{Glossary}

\section{Perforin}

A pore-forming protein that is stored in the cytolytic granules of CTLs and NK cells. It is essential for cytotoxicity.

\section{Granzymes}

Cytolytic granule-contained serine proteases that cleave substrates in the cytoplasm of target cells, triggering rapid apoptosis.

\section{Immune synapse}

The interface formed upon receptor-mediated recognition between an immune cell and antigen-presenting cell.

\section{Microtubule organizing centre (MTOC)}

A structure found in eukaryotic cells, where the minus ends of microtubules are anchored and from which the plus ends of microtubules extend. Cells can contain multiple MTOCs.

\section{The centrosome}

Usually consists of two centrioles surrounded by pericentriolar material and is the only MTOC in T cells.

\section{Artificial planar synapses}

Formed between $\mathrm{T}$ cells and lipid bilayers containing stimulatory ligands (for example, peptide-MHC complexes) supported on a cover slip.

\section{Partitioning defective (PAR) complex}

A conserved protein complex that regulates cell polarity in eukaryotes and consists of PAR3, PAR6 and an atypical protein kinase $C$ (such as PKC $)$ [Au:OK? PAR1B also mentioned in Figure 4?] .

\section{Pioneer microtubules}

These microtubules extend from the microtubule-organizing centre (such as the centrosome) to the leading edge of cells.

\section{Casein Kinase $1 \delta$ (CK1 $\delta$ )}

A serine/threonine kinase that mediates the phosphorylation of tubulin, microtubule nucleation and the phosphorylation of microtubule-associated proteins.

\section{Primary cilia}

Immotile single hair-like extensions from the plasma membrane formed by most cells that integrate signals from the environment.

\section{Intraflagellar transport (IFT)}

The bidirectional transport of protein complexes along microtubules in and out of the cilium or flagellum, which is required for ciliary assembly, resorption and signalling.

Transient receptor potential (TRP) channels

A large family of multifunctional ion channels, most of which are permeable to $\mathrm{Ca}^{2+}$.

Hedgehog signalling pathway 
An important signaling pathway for embryonic development and postnatal tissue maintenance in invertebrates and vertebrates. Mutations in the pathway can lead to cancer.

\section{Smoothened (SMO)}

A seven-pass transmembrane receptor and the key signal transducer in the Hh signalling pathway. 


\section{Key points}

- Ordering the events leading to formation of the cytolytic synapse provides a better understanding of the molecular mechanisms involved.

- The reorganization of both actin and microtubule cytoskeletons has key roles in polarizing secretion at the cytolytic synapse.

- The immune synapse and the primary cilium have structural and functional similarities; both structures provide sensing and signalling platforms in cells.

- Hedgehog signalling is associated with both primary cilia and immune synapses, which indicates that the immune synapse could have arisen from the evolutionary ancient primary cilium found in many organisms.

\section{Author biographies}

\section{Gillian Griffiths}

Gillian Griffiths is a Wellcome Trust Principal Research Fellow and Professor of Immunology and Cell Biology at the Cambridge Institute for Medical Research, UK. Her research focus is understanding the molecular mechanisms that control secretion at the immune synapse. http://www.cimr.cam.ac.uk/research/principal-investigators/principalinvestigators-a-h/griffiths

\section{Yukako Asano}

Yukako Asano is a research fellow with Gillian Griffiths at the Cambridge Institute for Medical Research, UK, specialising in live 4D imaging of immune cells.

\section{Maike de la Roche}

Maike de la Roche was a post-doctoral fellow in the Griffiths laboratory and is now a Sir Henry Dale Fellow and Junior Group Leader at the Cancer Research UK Cambridge Institute, where she is investigating the role of Hedgehog signalling in effector and memory function of T cells in vivo.

\section{Competing interests}

The authors declare no competing financial interests.

\section{Subject categories}

Biological sciences / Immunology / Imaging the immune system

[URI /631/250/2503]

Biological sciences / Immunology / Lymphocytes / T cells / CD8-positive T cells / Cytotoxic T cells 
[URI /631/250/1619/554/1834/1269]

Biological sciences / Immunology / Adaptive immunity / Cellular immunity [URI /631/250/2152/1566]

\section{Table of contents}

Structural and functional similarities between the cytolytic synapses formed by $\mathrm{CD}^{+} \mathrm{T}$ cells and primary cilia are shedding new light on the mechanisms and order of events of synapse formation. 